\title{
Algebraic model for scattering in three-s-cluster systems. II. Resonances in the three-cluster continuum of ${ }^{6} \mathrm{He}$ and ${ }^{6} \mathrm{Be}$
}

\author{
V. Vasilevsky, A. V. Nesterov, F. Arickx, and J. Broeckhove \\ Bogolyubov Institute for Theoretical Physics, Kiev, Ukraine \\ and Universiteit Antwerpen (RUCA), Department of Mathematics and Computer Science, Antwerp, Belgium
}

(Received 14 April 2000; published 14 February 2001)

\begin{abstract}
The resonance states embedded in the three-cluster continuum of ${ }^{6} \mathrm{He}$ and ${ }^{6} \mathrm{Be}$ are obtained in the algebraic version of the resonating group method. The model accounts for a correct treatment of the Pauli principle. It also provides the correct three-cluster continuum boundary conditions by using a hyperspherical harmonics basis. The model reproduces the observed resonances well and achieves good agreement with other models. A better understanding for the process of formation and decay of the resonance states in six-nucleon systems is obtained.
\end{abstract}

DOI: 10.1103/PhysRevC.63.034607

PACS number(s): 24.10.-i, 21.60.Gx, 25.70.Ef, 27.20.+n

\section{INTRODUCTION}

In this paper we investigate the three-cluster continuum of nuclei ${ }^{6} \mathrm{He}$ and ${ }^{6} \mathrm{Be}$, determined by the three-cluster configurations $\alpha+n+n$ and $\alpha+p+p$. This is a first calculation of the algebraic model (AM) for scattering in three-s-cluster systems, as described in Ref. [1]. Our objective is to highlight the quantitative and qualitative characteristics of algebraic model three-cluster calculations, as well as to produce accurate results for the astrophysically relevant resonances in ${ }^{6} \mathrm{He}$ and ${ }^{6} \mathrm{Be}$.

As the algebraic model for three-cluster scattering leads to a set of coupled-channel Schrödinger equations, we will transform the $S$ matrix to a diagonal form. This is usually referred to as the eigenchannel representation of the $S$ matrix [1]. We will derive the position and width of the resonances in the usual way, i.e., by scanning the corresponding eigenphase shifts $\delta$ as a function of energy for resonance behavior. To be precise, we determine position and widths through the conditions

$$
\left.\frac{d^{2} \delta}{d E^{2}}\right|_{E=E_{r}}=0, \quad \Gamma=\left.2\left[\frac{d \delta}{d E}\right]^{-1}\right|_{E=E_{r}} .
$$

Alternative methods have been suggested, such as the complex scaling method [2,3] (CSM). This method is based on a complex scaling of the Hamiltonian which reduces the scattering problem to an eigenvalue problem of a complex non-Hermitian Hamiltonian. The method was shown to reproduce efficiently both very narrow as broad resonance states. The drawback to the method is connected to the oscillatory behavior of the resonance. To get a stable and correct result for the position of the resonance, this behavior should be properly reproduced, requiring the calculation of huge complex Hamiltonian matrices. An interesting review, covering CSM as well as other methods for the determination of resonance characteristics, is presented in Ref. [4].

A third method of interest for evaluating the poles of the $S$ matrix called the continuation in the coupling constant (CCCM) and is appropriate for resonances near to the threshold [5]. A coupling parameter $\lambda$ is introduced such that for $\lambda=0$ repulsion in the system is suppressed, and for $\lambda=1$ the original system is recovered. The parameter $\lambda$ is varied from zero up to a critical value $\lambda_{c}$ at which value the bound state crosses the threshold; by analytic continuation up to $\lambda=1$ one then obtains a good approximation for a pole of the $S$ matrix in the complex energy plane.

In our work the nature and properties of the low-lying $0^{+}$ and $2^{+}$resonances in ${ }^{6} \mathrm{Be}$ and the $2^{+}$resonance state in ${ }^{6} \mathrm{He}$ will be studied in detail. The results of this paper are the next step in an investigation of six-nucleon systems in the threecluster model, initiated in Refs. [6,7]. In Refs. [6,7] we focused mainly on the problems of convergence and of the selection of the dominant part of the three-cluster subspace for the bound state properties in ${ }^{6} \mathrm{He}$ and ${ }^{6} \mathrm{Li}$. In the current paper we consider an appropriate mixture of basis functions tailored to describe both the internal and asymptotic regions. In particular, as the asymptotic behavior is reproduced most naturally by a hyperspherical harmonics (HH) description, the basis has to account for a sufficiently large set of hyperspherical harmonic wave functions. As already indicated above, the resonance position and widths will be obtained through the standard eigenphase analysis.

Several models and methods have already been applied to investigate the resonance states of ${ }^{6} \mathrm{He}$ and ${ }^{6} \mathrm{Be}$. In the series of papers [8-10], these systems were considered as threeparticle ones, and consequently antisymmetrization was neglected. These authors used an effective interaction between the alpha-particle and a nucleon, and a $N N$ realistic potential between the two nucleons. In these papers the hyperspherical harmonics method was used to describe the bound as well as the three-particle continuum states. In Ref. [11] the CSM was used to calculate the characteristic parameters of the resonance states in ${ }^{6} \mathrm{He}$ and ${ }^{6} \mathrm{Be}$. This was done within a three-cluster model, in which full antisymmetrization was taken into account, and the interactions between clusters was obtained from the Minnesota $N N$ potential. In Ref. [12] it was demonstrated that the method of continuation in the coupling constant (CCCM), used for the $\alpha+N+N$ configurations in ${ }^{6} \mathrm{He}$ and ${ }^{6} \mathrm{Li}$, reproduces results which are very close to those obtained by the CSM. We will compare our results to all three alternative methods. 


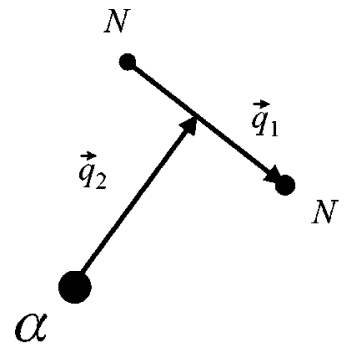

$4+2$

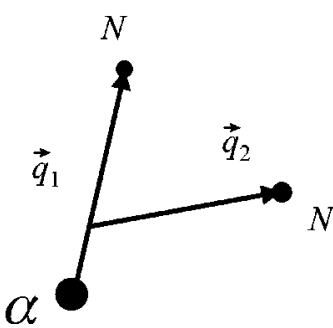

$5+1$

FIG. 1. Two configurations of the Jacobi coordinates for the three-cluster system $\alpha+N+N$.

\section{DETAILS OF THE CALCULATION}

To model the interaction between nucleons the Volkov potential [13] has been used throughout all calculations. As was shown in Refs. [6,7] it provides an acceptable description for ${ }^{6} \mathrm{He}$ within the three-cluster model. The effective Volkov potential consists of central forces only, so that total angular momentum $L$, total spin $S$, and total isospin $T$ are good quantum numbers. We will therefore consider only three-cluster configurations $\alpha+N+N$ with $S=0$ and $T=1$. This is known to be the most prominent and dynamically distinguished spin-isospin state for the resonances of interest in the nuclei considered in this work. The Coulomb interaction has also been included, as it is to a great extent responsible for reproducing the $0^{+}$resonance state in ${ }^{6} \mathrm{Be}$.

The oscillator radius $b$, associated with the basis functions for the nuclear state, is the only free parameter for the algebraic model calculation. We have chosen to fix $b$ by optimizing the ground-state energy of the $\alpha$ particle with respect to the Volkov interaction. This leads to a value of $b$ $=1.37 \mathrm{fm}$.

As explained in Ref. [1], we have a choice of Jacobi coordinate systems. This provides an additional degree of freedom that can be exploited to simplify the calculations. For a cluster configuration $\alpha+N+N$ one can consider the two Jacobi configurations displayed in Fig. 1. The first (referred to as the " $4+2$ " configuration) is the most appropriate for the current calculation. Selection rules effect a significant reduction in the number of basis functions. Indeed, quantum numbers $S=0, T=1$ coincide for the full six-nucleon system and the two-nucleon subsystem $(N+N)$. Hence the relative motion wave function must be an even function in the coordinate $q_{1}$ of this Jacobi system. This means that only even angular momenta $l_{1}$ have to be considered. Moreover, for positive parity states only even values of $l_{2}$, and for negative parity states only odd values of $l_{2}$ should be taken into account. With the second Jacobi configuration from Fig. 1 (the " $5+1$ ", one), these constraints are hard to meet and the full basis of oscillator states would have to be considered.

Table I shows the number of HH's $\left(N_{h}\right)$ of given hypermomentum $K$ for $L^{\pi}=0^{+}$and $L^{\pi}=2^{+}$in the " $4+2$ "' Jacobi configuration. This table also indicates the total number of HH's so far $\left(N_{c}\right)$ with $K=K_{\min }, K_{\min }+2, \ldots, K_{\max }$, i.e., it shows the number of channels for a given $K_{\max }=K$.

TABLE I. Enumeration of the number of hyperspherical harmonics $N_{h}$ for fixed value of $K$ and the accumulated number of hyperspherical harmonics $N_{c}$.

\begin{tabular}{cccccccc}
\hline \hline$L^{\pi}=0^{+}$ & $K$ & 0 & 2 & 4 & 6 & 8 & 10 \\
\hline & $N_{h}$ & 1 & 1 & 2 & 2 & 3 & 3 \\
& $N_{c}$ & 1 & 2 & 4 & 6 & 9 & 12 \\
\hline$L^{\pi}=2^{+}$ & $K$ & 0 & 2 & 4 & 6 & 8 & 10 \\
\hline & $N_{h}$ & & 2 & 3 & 5 & 6 & 8 \\
& $N_{c}$ & & 2 & 5 & 10 & 16 & 24 \\
\hline \hline
\end{tabular}

\section{A. Overlap matrix elements}

As full antisymmetrization between all nucleons is taken into account within the interaction region of the clusters, we first investigate the role of the Pauli principle for threecluster configurations. This is of importance amongst others for marking the boundary between the internal (interaction) region and the asymptotic region. No particle exchanges should occur in the asymptotic region. In the algebraic model the antisymmetrization effects can be visualized through the overlap and potential matrix elements. In resonating group method descriptions such as the integrodifferential or the generator coordinate versions, overlap and potential matrix elements are nonlocal functions of the coordinates, and are difficult to analyze. We use the notations of Ref. [1] for the overlap matrix elements, and in particular the shorthand notation $\nu_{0}$ is used for the set $\left(l_{1} l_{2}\right) L M$ of quantum numbers:

$$
\left\langle n,\left(K, \nu_{0}\right)|\hat{\mathcal{A}}| n^{\prime},\left(K^{\prime}, \nu_{0}\right)\right\rangle \text {. }
$$

Nonzero matrix elements (2) can be obtained from states within the same many-particle oscillator shell only. As the oscillator shells in the hyperspherical description are characterized by $N=2 n+K$, the selection rule becomes $2 n+K$ $=2 n^{\prime}+K^{\prime}$.

In Fig. 2 overlap matrix elements diagonal in $n$ for $L$ $=0$ and hypermomenta $K=0$ and $K=2$ are shown for the

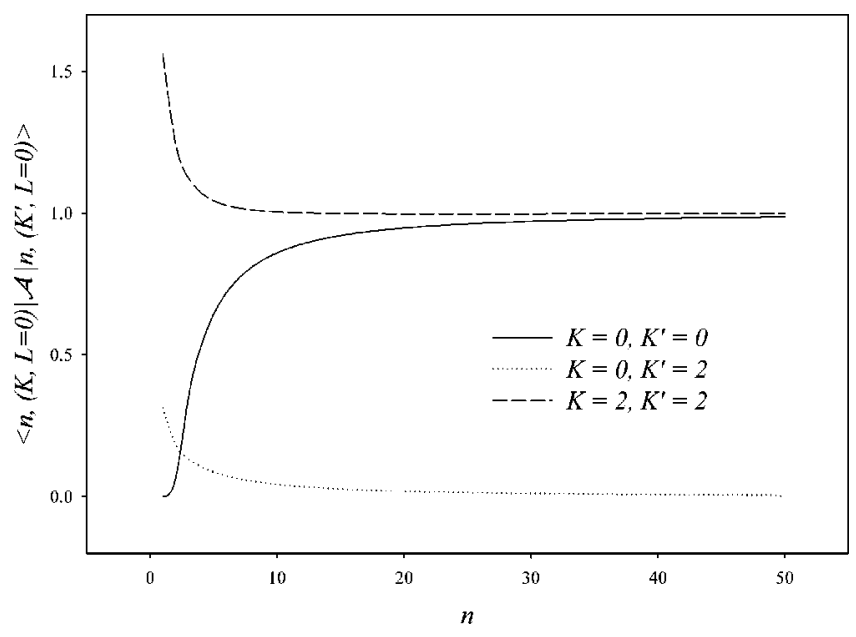

FIG. 2. Matrix elements $\left\langle n,(K, L=0)|\hat{\mathcal{A}}| n,\left(K^{\prime}, L=0\right)\right\rangle$ of the antisymmetrization operator for the $0^{+}$state in ${ }^{6} \mathrm{He}$ and ${ }^{6} \mathrm{Be}$. 


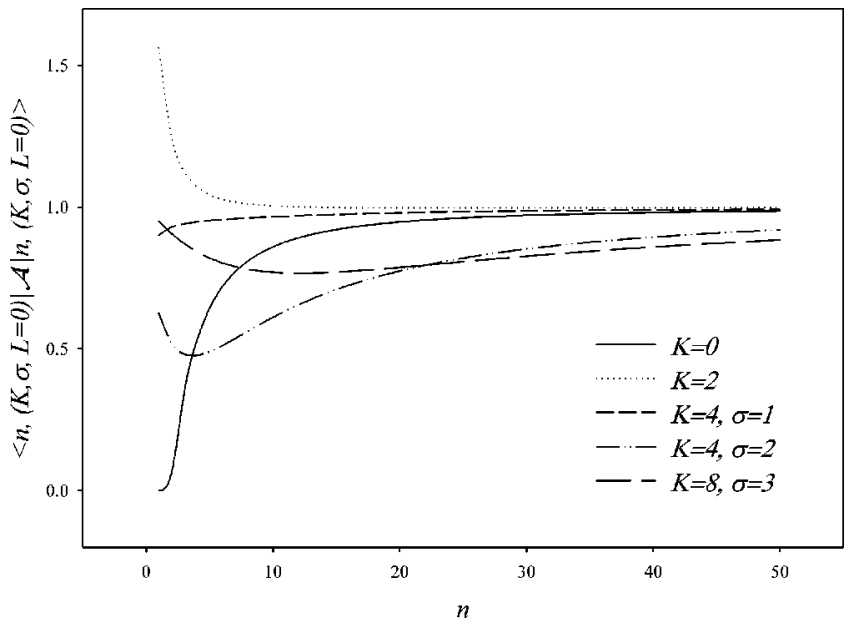

FIG. 3. Matrix elements $\langle n,(K, \sigma, L=0)|\hat{\mathcal{A}}| n,(K, \sigma, L=0)\rangle$ of the antisymmetrization operator for the $0^{+}$state in ${ }^{6} \mathrm{He}$ and ${ }^{6} \mathrm{Be}$.

six-nucleon three-cluster system. One notices from this figure that the Pauli principle involves oscillator states of at least the 25 lowest shells, for both diagonal and off-diagonal matrix elements in $K$. The antisymmetrization effects are visible in the deviation from unity for the diagonal matrix elements, and the deviation from zero of the off-diagonal (in $K$ ) elements. These effects decrease monotonically with higher $n$.

In Fig. 3 we compare matrix elements diagonal in $n$ and $K$ $\langle n,(K, \sigma, L=0)|\hat{\mathcal{A}}| n,(K, \sigma, L=0)\rangle$ for some of the $K$ values, $\sigma$ being a multiplicity quantum number for states with identical $K$. Only those matrix elements where the Pauli principle effect is most prominent have been shown. Some states with $K=4$ and $K=8$ are affected more strongly by antisymmetrization than others. To understand this we note that the Hyperspherical angles (corresponding to the hyperangular quantum numbers $\left.K, l_{1}, l_{2}, L M\right)$ define the most probable triangular shape and orientation in space of the three-cluster

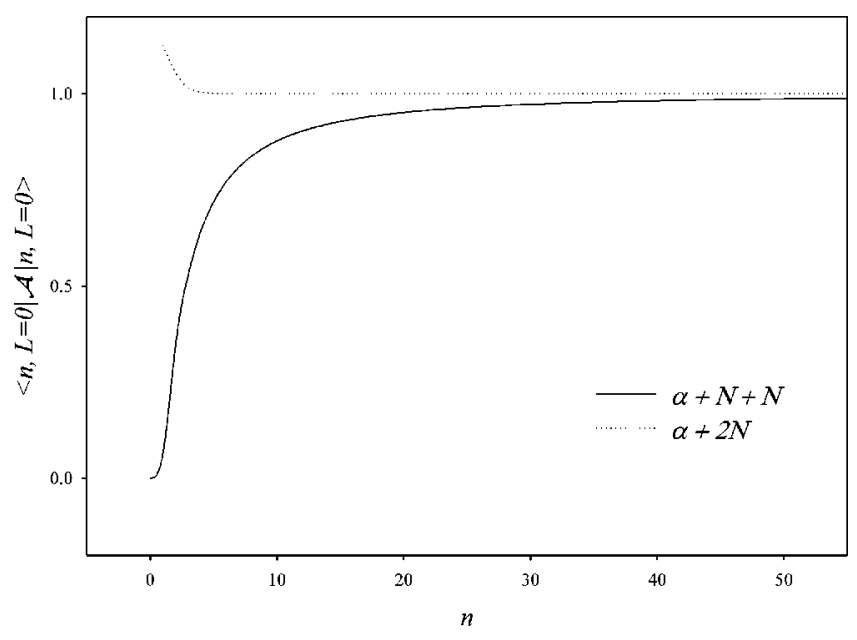

FIG. 4. Comparison of matrix elements of the antisymmetrization operator for the $0^{+}$state of ${ }^{6} \mathrm{He}$ and ${ }^{6} \mathrm{Be}$ obtained in the threecluster configuration $\alpha+N+N$ (for $K=0$ ) and the two-cluster configuration $\alpha+2 N$.

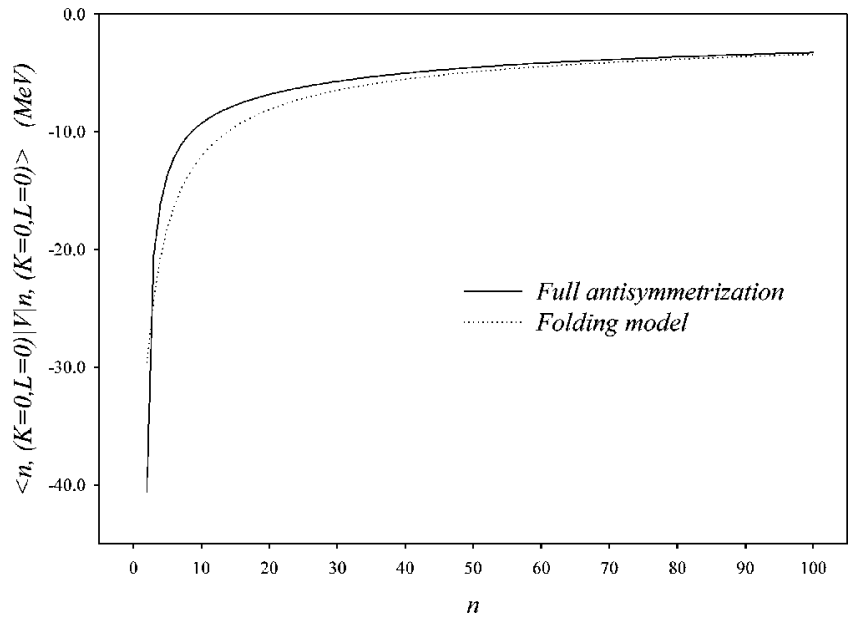

FIG. 5. Diagonal matrix elements of the potential energy operator for the $0^{+}$state of ${ }^{6} \mathrm{Be}$ and ${ }^{6} \mathrm{He}$ (for $K=0$ ) with full antisymmetrization compared to those within the folding model.

system. The HH's with $K=4, \sigma=2$ (characterized by $l_{1}$ $=l_{2}=2$ ) and $K=8, \sigma=3$ (characterized by $l_{1}=l_{2}=4$ ) seem to describe a triangular shape where one of the nucleons is very close to the $\alpha$ particle.

For larger $K$ values the probability to find all clusters close to one another within a hypersphere of fixed radius $\rho$ decreases, and one can expect that HH's with large values of $K$ will play a diminishing role in the calculations.

It is interesting to compare overlap matrix elements for the three-cluster configuration $\alpha+N+N$ with those of the two-cluster configurations $\alpha+2 N$ (such as $\alpha+d$ in ${ }^{6} \mathrm{Li}$ or $\alpha+2 n$ in $\left.{ }^{6} \mathrm{He}\right)$. This comparison is shown in Fig. 4, and it indicates that the Pauli principle has a much larger "range", in the three-cluster than in the two-cluster configuration.

As the kinetic energy in the algebraic model is made up of a product of the overlap and the three-particle kinetic energy, the antisymmetrization effects are identical to those of the overlap.

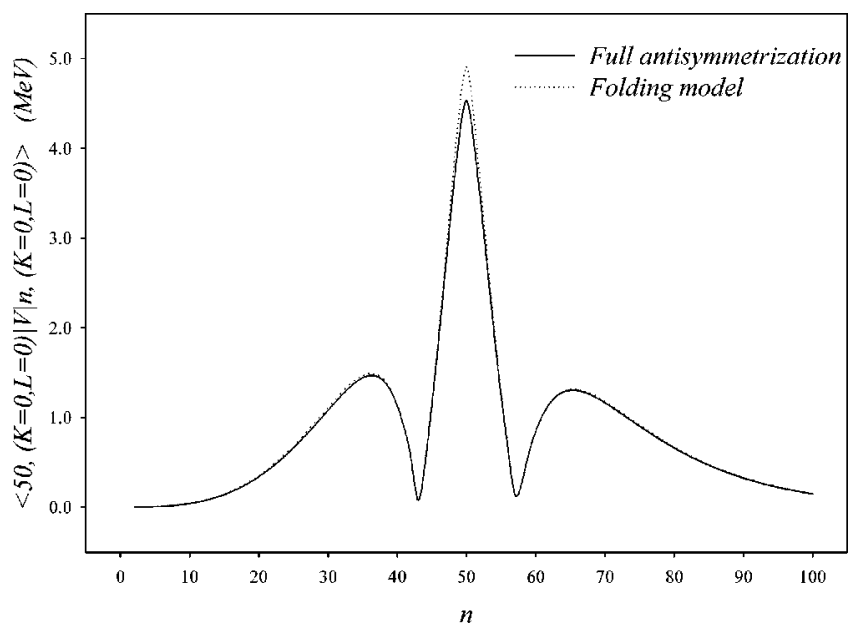

FIG. 6. Absolute value of off-diagonal matrix elements of the potential energy operator for the $0^{+}$state of ${ }^{6} \mathrm{Be}$ and ${ }^{6} \mathrm{He}$ (for $K$ $=0)$ with full antisymmetrization compared to those within the folding model. 


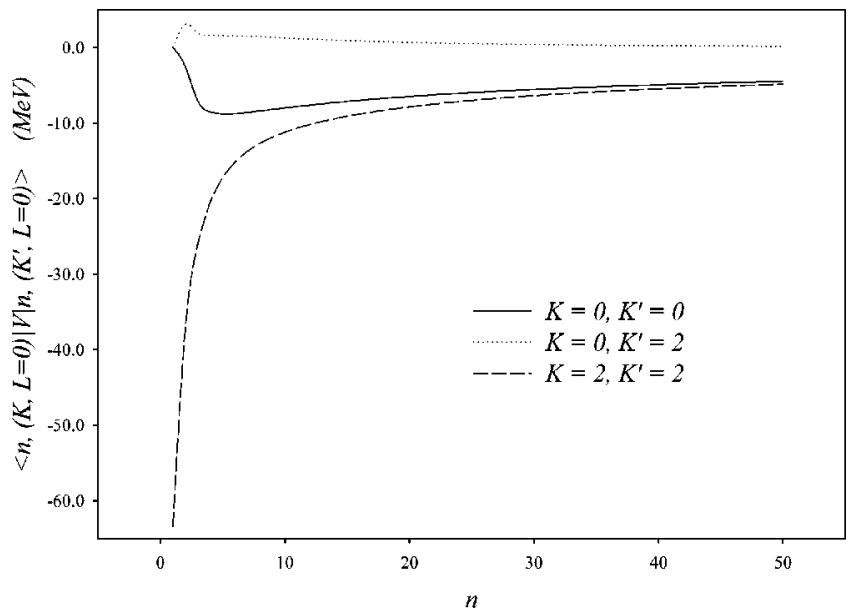

FIG. 7. Matrix elements of the potential energy operator for the $0^{+}$state of ${ }^{6} \mathrm{Be}$ and ${ }^{6} \mathrm{He}$, diagonal in $n$, with full antisymmetrization.

\section{B. Potential matrix elements}

To investigate the Pauli effect on the potential energy of the three-cluster configuration $\alpha+N+N$ we compare the potential matrix elements with hypermomentum $K=0$ with full antisymmetrization against those in the folding approximation, where antisymmetrization between clusters is neglected. Figure 5 shows the diagonal potential matrix elements $\left\langle n,\left(K=0, \nu_{0}\right)|V| n,\left(K=0, \nu_{0}\right)\right\rangle$ and Fig. 6 shows the matrix elements [actually the absolute value of, to eliminate a phase factor $(-1)^{n_{\rho}+n_{\rho}^{\prime}}=(-1)^{50+n}$ ) along a fixed row $\left\langle n=50,\left(K=0, \nu_{0}\right)|V| n,\left(K=0, \nu_{0}\right)\right\rangle$, calculated both with and without antisymmetrization between clusters. One notes that the folding model results are very close to the fully antisymmetrized ones, especially for larger $n$.

In Fig. 7 we also display the potential matrix elements between states of the two lowest values of hypermomentum $K=0$ and $K=2$. One sees that the $K=2$ contribution is the largest. The potential energy for $K=0$ is relatively small, and so is the coupling between $K=0$ and $K=2$ states.

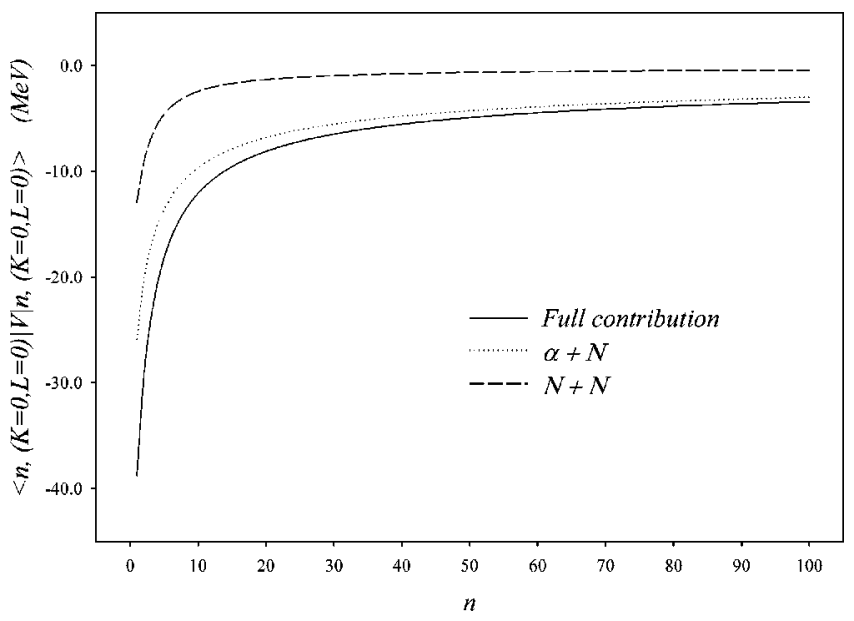

FIG. 8. Contributions in the folding model of the main components to the diagonal matrix elements of the potential energy operator for the $0^{+}$state of ${ }^{6} \mathrm{Be}$ and ${ }^{6} \mathrm{He}$ (for $K=0$ ).
TABLE II. Matrix of effective charges for the $0^{+}$state in ${ }^{6} \mathrm{Be}$.

\begin{tabular}{lrrrr}
\hline \hline$K ; l_{1}, l_{2}$ & $0 ; 0,0$ & $2 ; 0,0$ & $4 ; 0,0$ & \multicolumn{1}{c}{$4 ; 2,2$} \\
\hline $0 ; 0,0$ & 7.274 & 0.006 & -0.129 & 1.414 \\
$2 ; 0,0$ & 0.006 & 7.146 & -0.436 & 0.314 \\
$4 ; 0,0$ & -0.129 & -0.436 & 7.428 & -0.877 \\
$4 ; 2,2$ & 1.414 & 0.314 & -0.877 & 9.098 \\
\hline \hline
\end{tabular}

The main conclusion is that, in the asymptotic region, the " "exact" potential energy can be substituted with the folding approximation. It leads to a considerable reduction in computational effort. We are led to the following setup for threecluster calculations. In the internal region, consisting of states of the lower oscillator shells and with a large probability to find the clusters close to one another, the fully antisymmetrized potential energy is used. In the asymptotic region, where the average distance between clusters is large, we neglect antisymmetrization and use the folding model potential.

The folding model provides additional insight in the structure of the interaction matrix. It is well known that neither the $\alpha+n$ nor the $n+n$ interaction can create a bound state in the corresponding subsystems of ${ }^{6} \mathrm{He}$. Only a full threecluster configuration $\alpha+n+n$ contains the necessary conditions to create a bound state. Within the folding model the total potential energy is naturally split up in the individual contributions from the three interacting pairs: the $\alpha$ particle and the first neutron, the $\alpha$ particle and the second neutron and the neutron-neutron pair. The folding model thus allows for a comparison of the various contributions to the potential energy. For $K=0$ the first two contributions are identical, and we only have to consider the $\alpha+n$ and the $n+n$ pairs. Figure 8 shows the diagonal matrix element contribution of both components, and one notices that $\alpha+n$ represents the main contribution.

\section{The effective charge}

When Coulomb forces are taken into account, one needs to determine the effective charge in order to properly solve the algebraic model equations. The effective charge unambiguously defines the effective Coulomb interaction in each channel as well as the coupling between different channels. The effective charge also defines the solutions in asymptotic region through Sommerfeld parameter (see Ref. [1]). Using the approach suggested in Ref. [1] the effective charges for the $0^{+}$and $2^{+}$states of ${ }^{6} \mathrm{Be}$ were calculated. Part of the

TABLE III. Matrix of effective charges for the $2^{+}$state in ${ }^{6} \mathrm{Be}$.

\begin{tabular}{lrrrrr}
\hline \hline$K ; l_{1}, l_{2}$ & $2 ; 2,0$ & $2 ; 0,2$ & $4 ; 2,0$ & $4 ; 0,2$ & $4 ; 2,2$ \\
\hline $2 ; 2,0$ & 7.253 & 0.400 & -0.224 & 0.546 & -0.751 \\
$2 ; 0,2$ & 0.400 & 7.244 & -0.309 & -0.004 & -0.601 \\
$4 ; 2,0$ & -0.224 & -0.309 & 6.942 & -0.186 & 0.431 \\
$4 ; 0,2$ & 0.546 & -0.004 & -0.186 & 7.694 & -0.671 \\
$4 ; 2,2$ & -0.751 & -0.601 & 0.431 & -0.671 & 7.345 \\
\hline \hline
\end{tabular}


TABLE IV. Parameters of the $0^{+}$resonance in ${ }^{6} \mathrm{Be}$ as a function of $K_{\max }^{(a)}$ for fixed $K_{\max }^{(i)}=8$.

\begin{tabular}{lccccc}
\hline \hline$K_{\max }^{(a)}$ & 0 & 2 & 4 & 6 & 8 \\
\hline$E, \mathrm{MeV}$ & 1.434 & 1.314 & 1.304 & 1.298 & 1.292 \\
$\Gamma, \mathrm{MeV}$ & 0.075 & 0.082 & 0.084 & 0.085 & 0.087 \\
\hline
\end{tabular}

corresponding matrixes of $\left\|Z_{K}^{K^{\prime}}\right\|$ are displayed in Tables II and III, respectively. One notices that the diagonal matrix elements are much larger than the off-diagonal ones. This justifies the approximation [1] of disregarding the coupling of the channels in the asymptotic region.

It is interesting to compare the three-cluster effective charge with the effective charge in the two-cluster configuration. For the latter we can write

$$
Z=Z_{1} Z_{2} e^{2} \sqrt{\frac{A_{1} A_{2}}{A_{1}+A_{2}}},
$$

where $A_{1}$ and $Z_{1} \quad\left(A_{2}\right.$ and $\left.Z_{2}\right)$ are the respective mass and charge of both clusters. For the configuration $\alpha+2 p$ in ${ }^{6} \mathrm{Be}$, we then obtain an effective charge

$$
Z=\frac{8}{\sqrt{3}} e^{2} \simeq 6.65
$$

which is independent on the angular momentum of the system. One notices that the two-cluster effective charge is close to the diagonal matrix elements of $\left\|Z_{K}^{K^{\prime}}\right\|$. We can assume that, if in one of the three-cluster channels the effective charge is very close to the two-cluster one, it could indicate that the two protons move as an aggregate in the asymptotic region. For the $2^{+}$state we observe at least one channel with this property, carrying the labels $K=4, l_{1}=2, l_{2}=0$.

\section{RESULTS}

\section{A. Definition of the model space}

The model space for the current calculations is primarily determined by the total number of HH's in the internal and external region, and the number of oscillator states. Different sets of HH's can be used in the internal and asymptotic regions. An extensive set of HH's in the internal region will provide a well correlated description of the three-cluster system due to the coupling between states with different hypermomentum. The HH's in the asymptotic region, which are exactly (without Coulomb) or nearly exactly (Coulomb included) decoupled, are responsible for the richness in decay possibilities.

TABLE V. Parameters of the $0^{+}$resonance in ${ }^{6} \mathrm{Be}$ as a function of $K_{\max }^{(a)}$ for fixed $K_{\max }^{(i)}=10$.

\begin{tabular}{lcccccc}
\hline \hline$K_{\max }^{(a)}$ & 0 & 2 & 4 & 6 & 8 & 10 \\
\hline$E, \mathrm{MeV}$ & 1.324 & 1.204 & 1.192 & 1.184 & 1.176 & 1.172 \\
$\Gamma, \mathrm{MeV}$ & 0.068 & 0.069 & 0.071 & 0.071 & 0.073 & 0.072 \\
\hline \hline
\end{tabular}

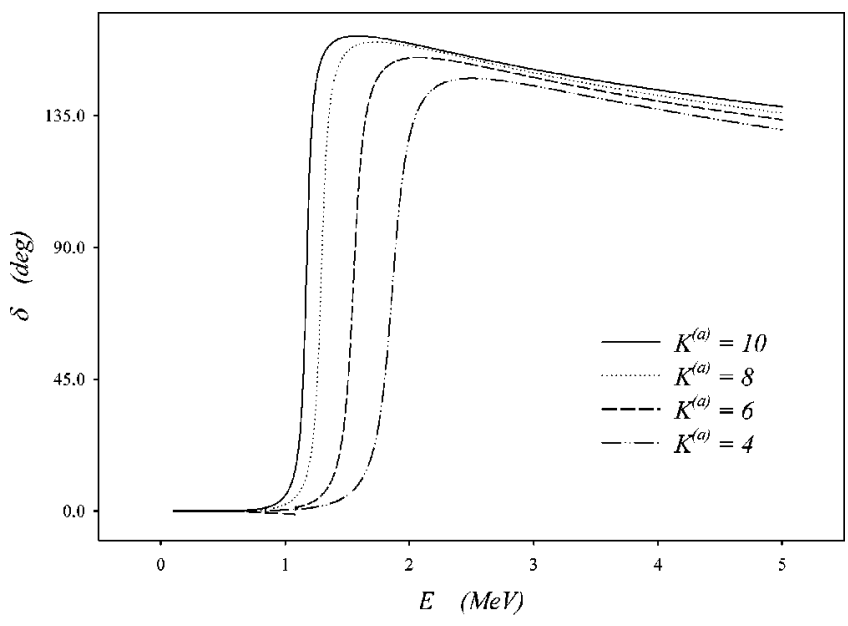

FIG. 9. Eigenphase shifts for the $0^{+}$state of ${ }^{6} \mathrm{Be}$ for different values of $K_{\max }^{(a)}$.

In the current paper we restricted both internal and asymptotic $\mathrm{HH}$ sets maximal hypermomentum value $K_{\max }^{(i)}$ $=K_{\max }^{(a)}=10$. By extending the respective subspaces up to these maximal values, we obtain a fair indication of convergence.

We fixed the matching point between internal and asymptotic region at $N=50$. It is based on the observations of the previous sections, i.e., (i) it is sufficiently large to allow the Pauli principle its full impact, and (ii) it is such that only possibly long-range effects of the potential are still remaining in the asymptotic region, which can then be taken properly into consideration in the defining three-term recursion formula for the asymptotic expansion coefficients of the algebraic model [1] . The choice of matching point thus represents a proper compromise between kinematical (i.e., Pauli) and dynamical (i.e., interaction) effects.

The resonance parameters are obtained from the eigenphase shifts obtained from the eigenchannel representation (diagonalized form) of the $S$ matrix.

\section{B. Convergence study}

We first consider the influence of the number of asymptotic channels. We do this by calculating the position and width of the $0^{+}$state in ${ }^{6} \mathrm{Be}$ using successively larger asymptotic subspaces. The first calculation includes all HH's up to $K_{\max }^{(i)}=8$ and extends the asymptotic subspace from $K_{\max }^{(a)}=0$ to $K_{\max }^{(a)}=8$. The second calculation includes all HH's up to $K_{\max }^{(i)}=10$ and extends the asymptotic subspace from $K_{\max }^{(a)}=0$ to $K_{\max }^{(a)}=10$. The corresponding results are shown in Tables IV and V. One learns from these results that a sufficient rate of convergence has been obtained, by which

TABLE VI. Parameters of the $0^{+}$resonance in ${ }^{6} \mathrm{Be}$ as a function of $K_{\max }^{(i)}$ for $K_{\max }^{(a)}=0$.

\begin{tabular}{lcccccc}
\hline \hline$K_{\max }^{(i)}$ & 0 & 2 & 4 & 6 & 8 & 10 \\
\hline$E, \mathrm{MeV}$ & 2.408 & 2.020 & 1.688 & 1.434 & 1.324 \\
$\Gamma, \mathrm{MeV}$ & 0.147 & 0.129 & 0.097 & 0.075 & 0.068 \\
\hline \hline
\end{tabular}




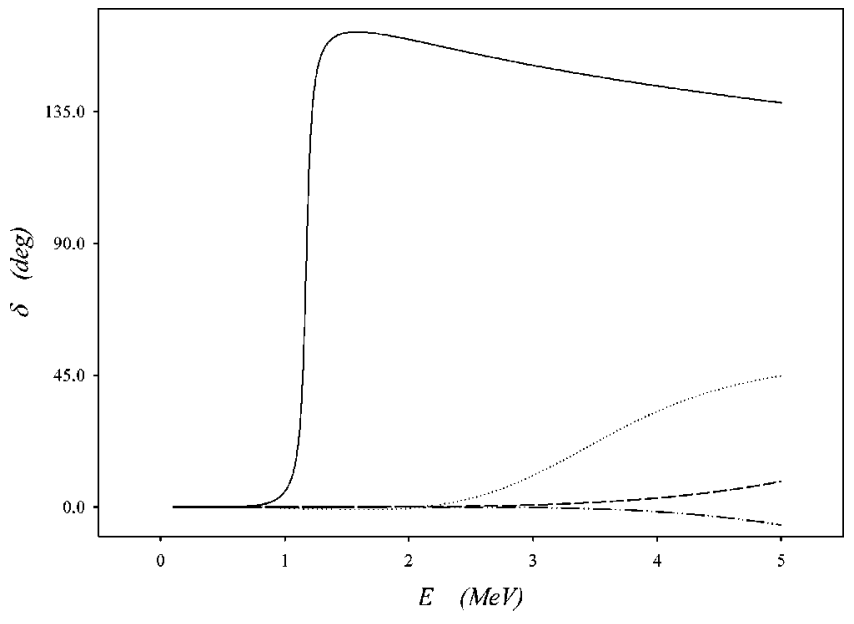

FIG. 10. The eigenphases for the $0^{+}$state in ${ }^{6} \mathrm{Be}$.

we mean that the qualitative behavior of the phase shifts and resonance parameters remains unchanged. Figure 9 displays the first eigenphase shift as a function of energy for $K_{\max }^{(i)}$ $=10$ and a choice of $K_{\max }^{(a)}$ values from which results in the tables are derived.

Whereas the inclusion of higher hypermomenta in the asymptotics shows a fast and monotonic convergence, it is also clear from these results that a sufficient number of HH's has to be used for a correct description of the correlations in the internal state. To support this conclusion, we performed a calculation, again for the $0^{+}$state in ${ }^{6} \mathrm{Be}$, in which only one $\mathrm{HH}$ with value $K^{(a)}=0$ was used. In the internal region the number of HH's was varied from $K^{(i)}=0$ up to $K^{(i)}=10$. These results appear in Table VI and corroborate the previous conclusion. In particular they indicate that the effective potential obtained with the $K^{(i)}=0 \mathrm{HH}$ only, is unable to produce a resonance. Only after including a $K=2 \mathrm{HH}$ does the resonance appear, with an overestimated energy and width. Further inclusion of higher $\mathrm{HH}$ states then lead to an acceptable convergence in a monotonically decreasing fashion for both position and width of the resonance.

The current calculations were limited to $K=10$ to reduce the numerical effort which is already high.

\section{Comparisons}

In Fig. 10 the shape is displayed of the (first few) eigenphase shifts for $L^{\pi}=0^{+}$in ${ }^{6} \mathrm{Be}$ in the full calculations, i.e. with the maximal number of internal and asymptotic HH's. One notices that the first $0^{+}$resonance state of ${ }^{6} \mathrm{Be}$ appears in the first eigenchannel, and that a second (broad) resonance at a higher energy is created in the second eigenchannel (see Table VII for details on this resonance). The phase shifts in the higher eigenchannels show a smooth behavior as a function of energy without a trace of resonances in the energy range that we consider.

Table VIII compares the results of this work to the experimental data available from Ref. [14], and to results obtained in other calculations, in particular CSM [11], HHM [9], and CCCM [12]. The agreement with the experimental energy and width of the resonant states is reasonable. The difference between the experimental and calculated energies of the $2^{+}$ resonance states in both ${ }^{6} \mathrm{He}$ and ${ }^{6} \mathrm{Be}$ are probably due to the lack on LS forces in the present calculations.

It has been pointed out in Ref. [9] as well as in Ref. [11], that the barrier created by the three-cluster configuration is sufficiently high and wide to accommodate two resonance states, the second one of which is usually very broad. At first sight, this could be ascribed to an artifact of the $\mathrm{HH}$ model since large values of hypermomentum $K$ create a substantial centrifugal barrier. However, the CSM calculations $[15,16]$, which do not use HH's, also reveal such resonances. Our calculations now also confirm the existence of a second very broad resonance. A comparison of the resonance parameters with those of the HH method [9] and of the CSM [11] is shown in Table VII. The differences are most probably due to the different descriptions of the system, as well as to the difference in $N N$ forces.

\section{CONCLUSION}

In this work we have presented the results of the lowenergy continuum spectrum of ${ }^{6} \mathrm{He}$ and ${ }^{6} \mathrm{Be}$ as obtained in the three-cluster description $\alpha+N+N$ within the algebraic model developed in Ref. [1].

It was shown that the range of the Pauli principle in threecluster systems extends well beyond the range in two-cluster models. This can be understood by the fact that, even at high values of the hyper-radius $\rho$, there is a nonzero probability for one pair of clusters to be close to one another, and thus within the range of the Pauli principle.

It was also shown that the folding approximation is already close to the fully antisymmetrized potential for relatively compact three-cluster configurations. Thus the folding approach, which is numerically much easier, can approximate the exact potential with good precision in a large part of the model space.

We have elaborated upon the role of the different $\mathrm{HH}$ states in the formation and decay of resonance states in the three cluster continuum, and have demonstrated that a suffi-

TABLE VII. Energy and width of the second resonance states, obtained by AM, HHM, and CSM.

\begin{tabular}{lccccccc}
\hline \hline Nucleus & $L^{\pi}$ & \multicolumn{2}{c}{ AM } & HH [9] & \multicolumn{3}{c}{ CSM [15,16] } \\
\hline & & $E, \mathrm{MeV}$ & $\Gamma, \mathrm{MeV}$ & $E, \mathrm{MeV}$ & $\Gamma, \mathrm{MeV}$ & $E, \mathrm{MeV}$ & $\Gamma, \mathrm{MeV}$ \\
${ }^{6} \mathrm{He}$ & $0_{2}^{+}$ & 2.1 & 4.3 & 5.0 & 6.0 & 3.9 & 9.4 \\
${ }^{6} \mathrm{He}$ & $2_{2}^{+}$ & 3.7 & 5.0 & 3.3 & 1.2 & 2.5 & 4.7 \\
${ }^{6} \mathrm{Be}$ & $0_{2}^{+}$ & 3.5 & 6.1 & & & & \\
${ }^{6} \mathrm{Be}$ & $2_{2}^{+}$ & 5.2 & 5.6 & & & & \\
\hline \hline
\end{tabular}


TABLE VIII. Energy and width of the resonace states in ${ }^{6} \mathrm{He}$ and ${ }^{6} \mathrm{Be}$, obtained by AM, HHM, CSM, and CCCM.

\begin{tabular}{lcccccc}
\hline \hline & \multicolumn{2}{c}{${ }^{6} \mathrm{He} ; L^{\pi}=2^{+}$} & \multicolumn{2}{c}{${ }^{6} \mathrm{Be} ; L^{\pi}=0^{+}$} & \multicolumn{2}{c}{${ }^{6} \mathrm{Be} ; L^{\pi}=2^{+}$} \\
\hline Method & $E, \mathrm{MeV}$ & $\Gamma, \mathrm{MeV}$ & $E, \mathrm{MeV}$ & $\Gamma, \mathrm{MeV}$ & $E, \mathrm{MeV}$ & $\Gamma, \mathrm{MeV}$ \\
Experiment [14] & $0.822 \pm 0.025$ & $0.133 \pm 0.020$ & 1.371 & $0.092 \pm 0.006$ & $3.04 \pm 0.05$ & $1.16 \pm 0.06$ \\
AM & 1.490 & 0.168 & 1.172 & 0.072 & 3.100 & 0.798 \\
HHM [9] & 0.75 & 0.04 & & & & \\
CSM [11] & 0.74 & 0.06 & 1.52 & 0.16 & 2.81 & 0.87 \\
CCCM [12] & 0.73 & 0.07 & & & & \\
\hline \hline
\end{tabular}

cient number of HH's should be considered for a proper description of the internal state. It was also seen that the number of HH's in the asymptotic region is of lesser importance. It was demonstrated that the $\mathrm{HH}$ basis thus represents an appropriate and convenient tool for describing threecluster systems, as it takes into account the proper boundary conditions for the continuum of multicluster configurations.

The calculated values for energy and width of the resonance states agree well with the observed experimental data. The results of the present model are also in good accordance with those obtained in alternative models used for the description of resonance states in six-nucleon systems.

The main conclusion to be drawn from this work is that the AM for three-cluster configurations, which takes into ac- count full antisymmetrization between all particles, not only leads to meaningful results, but provides a feasible alternative to other models.

\section{ACKNOWLEDGMENTS}

This work was partially supported by an INTAS Grant ("INTAS-93-755-extension"). One of the authors (V.V.) gratefully acknowledges the University of Antwerp (RUCA) for a "RAFO-gastprofessoraat 1998-1999" and the kind hospitality of the members of the research group Computational Quantum Physics of the Department of Mathematics and Computer Sciences, University of Antwerp, RUCA, Belgium.
[1] V. S. Vasilevsky, A. V. Nesterov, F. Arickx, and J. Broeckhove, Phys. Rev. C 63, 034606 (2000), preceding paper.

[2] J. Aguilar and J. M. Combes, Commun. Math. Phys. 22, 269 (1971).

[3] E. Balslev and J. M. Combes, Commun. Math. Phys. 22, 280 (1971).

[4] Y. K. Ho, Phys. Rep. 99, 1 (1993).

[5] V. M. Krasnopol'sky, V. I. Kukulin, and J. Horacek, Theory of Resonances. Principles and Applications (Kluwer Academic, Dordrecht, 1989).

[6] F. Arickx, P. Van Leuven, V. S. Vasilevsky, and A. V. Nesterov, in New Perspectives in Nuclear Structure, Proceedings of the 5th International Spring Seminar in Nuclear Physics, Ravello, Italy, 1995, edited by A. Covello (World Scientific, Singapore, 1996), p. 111.

[7] V. S. Vasilevsky, A. V. Nesterov, F. Arickx, and P. Van Leuven, Yad. Fiz. 60, 413 (1997) [Phys. At. Nucl. 60, 343 (1997)].
[8] M. V. Zhukov, B. V. Danilin, D. V. Fedorov, J. M. Bang, I. J. Tompson, and J. S. Vaagen, Phys. Rep. 231, 151 (1993).

[9] B. V. Danilin, M. V. Zhukov, S. N. Ershov, F. A. Gareev, R. S. Kurmanov, J. S. Vaagen, and J. M. Bang, Phys. Rev. C 43, 2835 (1991).

[10] B. V. Danilin, T. Rodge, S. N. Ershov, H. Heiberg-Andersen, J. S. Vaagen, I. J. Tompson, and M. V. Zhukov, Phys. Rev. C 55, R577 (1997).

[11] A. Csoto, Phys. Rev. C 49, 3035 (1994).

[12] Y. Suzuki, N. Tanaka, and K. Varga, Phys. Rev. C 56, 562 (1997).

[13] A. B. Volkov, Nucl. Phys. 74, 33 (1965).

[14] F. Ajzenberg-Selove, Nucl. Phys. A490, 1 (1988).

[15] S. Aoyama, S. Mukai, K. Kato, and K. Ikeda, Prog. Theor. Phys. 93, 99 (1995).

[16] S. Aoyama, S. Mukai, K. Kato, and K. Ikeda, Prog. Theor. Phys. 94, 343 (1995). 